\title{
Reasons for a Cultural Renovation of the Road Infrastructure Design
}

\author{
Alfonso Annunziata and Francesco Annunziata \\ Department of Civil And Environmental Engineering and Architecture, University of Cagliari, Cagliari I-09125, Italy
}

\begin{abstract}
Built environments are undergoing a state of crisis, due to fragmentation and obsolescence: consolidated urban areas, suburbs and fringe areas require to be restored so as to recover a sense of vivid places and to avoid segregation. Fabric of routes and voids can be considered as a medium in order to structure a landscape, to manage and govern a region, to nurture its unique subculture. A structure of bonds is to be designed to promote a rapid and safe movement of resources, of people, of ideas, even from and to most remote sites. Infrastructure can foster a vast economic development, and also, a profound social and cultural development.
\end{abstract}

Key words: Adaptation, infrastructure, landscape, management, public space, interactions.

\section{Introduction}

Tendencies of super-urbanization [1], due to rise and development of urban economies and increase in scope and magnitude of streams of resources, people, ideas, dross, capitals engaged in a more and more broad and rapid urban metabolism, can represent a strain for built environments: increase of population, social segregation, required variations of aims and scope of policies, cultural ferments and erosion of ecological structures, depletion of rural areas, congestion, soil consumption beside climate perturbation, scarcity of resources, calamitous events and failures and obsolescence of infrastructure are going to burden cities and adjacent regions.

As a consequence, ecological concerns, preservation of rural areas, a demand for softer economies, so as scope and magnitude of interactions engaged in urban routines, impose a diverse urban model and diverse forms of governance. The aim is to repair a profound fracture among permanence of built environments, routines of public agencies, paces of

Corresponding author: Alfonso Annunziata, Ph.D., research fields: landscape infrastructure, renovation of urban spaces and of edges and residual spaces generated by transport infrastructures. E-mail: annunziata.alfonso@yahoo.it. evolution of socio-economic tendencies [2], but also a fracture among economies and ecologies, among scope and influences of urbanism and bureaucratic boundaries, among infrastructure and social, ecological, spatial factors of traversed sites [3].

Infrastructure, as a device meant to favor streams of resources and users, required in order to enable urban practices, individuates belts of land apt to sustain most valuable urban features and land uses. As a result, it is to be conceived in order to define spatial models founded on a balanced distribution of functions and meant to reduce scope and outcomes of resource streams, to prevent an ominous consumption of scarce resource (for instance, fertile soils), to moderate burdens on environment. As a consequence, a diverse notion of infrastructure (and of management of infrastructure) is to be proposed: a rigid and bureaucratic management and design of transport infrastructure, based on utilitarian parameters, reliant on a clear separation of diverse provisions and modes of use [4], on a net negation of sites and on a reduction of regions to a list of use-values, in order to ensure control and economic convenience, is to be replaced by a model of governance based on an encounter of diverse actors and instances so as to 
engage diverse levels and forms of perception of a given region. As observed by Hung [5], "the trend for today's infrastructure system is to become decentralized, where the need to address...stormwater runoff, energy, farming or transportation are resolved at a local level”. Inclusion, as a mean for protection and preservation of a region and of its core of sense, instead of number of potential users and estimated revenues, becomes a main parameter.

\section{Aims of Conversion and Upgrade of Infrastructure}

Infrastructure is to be conceived as a frame for broader urban regions, for mosaics of denser urban fabrics, farmlands, production poles, ecotopes, open spaces, meant to repair to a sterile divide amid rural and urban, to favor osmosis among land uses, a concordance among distribution of resources, ecological factors and land uses, to enable circular softer economies and urban practices founded on reuse and conversion of residues and dross and able to protect and engage traditions, social and cultural values, and ecological forces.

Renovation, conversion and reuse of infrastructures are to be considered as opportunities to remediate, regenerate and transform urban realms. Restore actions presume an upgrade of infrastructures, a diversification of modes of transport and urge to consider and set a relation among scale of routes, purpose and scope of movements and land uses or routines located along edges. An organic, soft, intermodal structure, founded on several levels and on a strict integration of diverse fabrics of routes, media and modes of transport is required: it is essential to favor concatenation among various transfers, or among various stages of a same transfer. A Movement from a point $A$ to a point $B$ is to be conceived, for instance, as a series of stages, of specific scale and served by a specific mode of transport and by a specific frame of routes of adequate level. Connections among diverse stages need to be rapid: pauses and stops must be reduced and transit among different routes or modes of transport is supposed to be prompt and simple.

Roads and transport lines can be recovered and re-used for movements diverse in scale and content and as routes for various modes of transport. Moreover, as a continuous space of more intense content, due to sequences of services and activities along its edges, infrastructure can represent a fecund provider of open spaces: a disused railroad may become a track for tram or light rail lines or as in the case of the Promenade plantée in Paris or of the Highline in New York City, designed by James Corner Field operations, it can be converted into a pedestrian promenade and become a spine of a vivid linear urban scenario.

\section{Transport Systems}

It is proper to focus not on urban areas, considered as realms defined by precise administrative boundaries, but on streams and interactions, involved in urban metabolism, so as to individuate a broader area of influence, a region, to be considered as a promising field of action for softer and sensitive policies. Regions are to be considered as macro areal entities, defined as a plot of interactions, due to a specific profound osmosis among landscapes and people, and composed of a structure of local entities, of social molecules, as stated by Olivetti [6]. As a consequence, in order for movement to mold a mosaic of diverse scenarios and land uses so as to avoid urban areas spreading into desolate suburbs, to avoid remote sites being depleted and abandoned and unique customs and rites being lost, transport fabrics, and in particular, roads, are to be merged in an organic system meant to serve even remotest points: therefore minor routes are to be considered as a valuable tool in order to sustain and to nurture a region.

Swiss railway systems appears to appropriate the concept of a broad structure articulated in a dense tissue of ties and nodes, inflected on various levels of 
scale, so as to serve most remote areas and to sustain and reinforce local life. Colin Ward observed that "the Swiss railway system is the densest network in the world. At great cost and with great trouble, it has been made to serve the needs of the smallest localities and most remote valleys, not as a paying proposition, but because such was the will of the people. The distribution of industrial activity all over Switzerland, even in the outlying areas, accounts for the strength and stability of the social structure of the country and prevented those horrible concentration of industry, with their slums and rootless proletariat (in largest urban areas)" [7].

\section{New Forms of Management}

Relations among transport structures, among transport devices and landscapes and a concept of inclusion of served realms are parameters and a main motive for a unified vision. It is proper to consider consequences, due to a profound transformation of a specific road, on transport fabrics and on land uses and landscapes: plans, design, actions of reuse, regeneration and upgrade, form a series of tied steps in a process of management, and emanate from actions of management of a broader fabric and, on a superior level, of the transport system. Moreover, a rigorous management implies to adapt a road to a set of performance standards, deduced from its function inside a specific transport frame and inside a specific region. Moreover, a diverse conception of roads as landscape re-generator, and of strategies and aims of management, leads to a diverse conception of functions to be implemented.

An advanced form of management starts from an analysis meant to define a fabric of spatial bonds needed to nurture uses and routines of a region.

The first step is an analysis of the demand for access to services, functions, major nodes of transport and users bases, advanced by a specific community. As a result, a set of required movements is defined. An order among required movements is given by relevance of individuated nodes or services.

Relevance depends on estimated size of user bases, or on estimated outcomes of a more rapid access to a specific service or function, in terms of an improvement of condition of life, of an increase of uses and activities, in a reduction of conditions of segregation.

In order to enable rapid and safe movements, to avoid segregation of more vulnerable social groups or users, to reduce depletion and erosion of ecological structures and of land uses and to prevent congestion, it is proper to deter and moderate car transit: as a consequence, it is proper to reduce number, scope and volume of movements, i.e., to reduce the transport demand, and to reinforce and foster softer forms of motion.

Medium scale functions can be implemented at local level and distributed across a region. A strong suture among local entities is required, in order to promote safe and rapid transfers. For major urban services, resources and transport nodes are to be recognized nearest poles of major scope.

A subsequent step must focus on definition, for main residual required movements, of proper modes of transport, of end points, scale, reasons, nature, sort of users and type of related nodes.

A main concern is to individuate as favored responses to residual needs, modes of transport alternative to cars. As a result, required transport functions, i.e., a precise demand for a specific set of connections is defined.

A subsequent step is based on a census of available transport devices in the given area. The desired outcome is the definition of a proper function for routes and infrastructures present in-situ. A first step focuses on individuation of corridors [8], i.e., of bundles of transport media serving a certain couple of end points. Moreover, it is due and proper to consider the dual nature of a road: roads are entities defined by a transport status, due to its size and measure, and related to a large scale. Roads also refer to a place 
status, focused on a local scale [8]. It is due and proper to evaluate a road in terms of its relevance inside an organic transport fabric, and in terms of its relevance as a place and as a relational device inside a specific realm. A main concern for a profound renovation of a road is to consider infrastructure as a landscape, a sequence of spaces apt to substitute usual urban forms as scenarios of various practices and functions [9]. As stated by Smets and Shannon [10], "once married with architecture, mobility and landscape, infrastructure can integrate territories more meaningfully, reduce marginalization and segregation, and stimulate new forms of interactions".

As a result, a first step must focus on scale of routes, on number of properties and area served by a specific road, in order to deduce its transport purpose. A second stage is meant to compare road section and side spaces to a set of standard sections, i.e., models of road related to specific purposes. As a consequence, a potential transport function, i.e., a potential connection status, is defined.

A second step must focus on traversed sites, and must tend to recognize land uses, sacred sites or references, so as to deduce, of each section of a road, a specific place status, described by scope, relevance and purposes, of a given segment and of its edges as a space for a vast set of uses and routines, related to uses and contents of traversed sites.

It is proper to notice that the relevance of a specific place status does not depend on its scope, i.e., on scale of land uses along its edges, but depends on presence of sensitive social, cultural, economic, ecological features, and on the consequent need of spaces conceived as a border and as a realm apt to preserve, order, sustain and nurture traversed sites.

The place status defines scale, relevance and nature of modes of use and interactions to be sustained along edges. It is proper to note that if the transport status of a road is constant along its course, its place status reveals a variation consonant in its pace and tones, to sequences of land uses and of relevant sites. Voids and fringe areas are to be considered as a field of action in order to confer a vaster sense to roads: public spaces, nodes, spots, open to diverse uses and diverse forms of motion, ecotopes, surfaces and devices conceived to capture, drain and store water, farmlands, areas and devices conceived to produce energy, can structure a various, multi-leveled space, apt to merge social factors, economic trends and ecological concerns.

A comparison among potential transport functions and a latent vocation as urban space is required in order to set a corpus of proper standards related to transport performances, promotion along road edges of social and spontaneous episodes and interaction among rapid transfers, practices along road edges and land uses present in traversed places.

Sites rich of unique or frail uses and contents, (dense residential areas, antique urban centers, major ecological structures and farmlands) are to be considered sensitive areas. As a consequence, if a road traverses a sensitive area, its place status is preponderant and it is to be considered prevalent on its potential transport function. A required convergence among moderation of costs, maintenance of performance levels and fruition of road spaces as vivid urban scenarios, is imposed to adopt measures of modulation of motion along a road [11], as a medium apt to convert fringes and transition spaces in a modulated version of an urban scenario, a space apt to favor events, diverse modes of use and contacts among people in specific areas and in precise periods. Modulation of nature, magnitude and outcomes on traversed sites of interactions, alternation of motion and social episodes and segregation of most dangerous forms of movement, are measures apt to decline the concept of modulation as an operative tool in order to merge motion and urban space.

A subsequent step focuses on a comparison among spaces required for implementation of demanded performance standards, road spaces and available residual border spaces, i.e., surfaces usable to create 
public space, to implement measures apt to preserve people realms and land uses and to increase transport performances. As a result, a precise function, i.e., a precise set of functions and of performance standards, is defined for each road to be re-used. A vast procedure of evaluation of roads and arcs present in a given area is focused on a precise definition of a set of targets to be posed as frame and reference for a profound revision of transport fabrics.

Above-mentioned models refer to Level 3 of a four levels categorization as proposed by Eppell et al. [12]. A subsequent stage consist in a comparison among transport functions insurable by the transport fabric of a region and transport functions required in order to serve its routines. In case a required set of performance standards, related to a given function, cannot be satisfied by the upgrade of a specific road, it could be considered to distribute the streams of users and resources among several routes of a same corridor, in order to reduce, on each road, spaces reserved for cars and burdens on traversed sites. The management of a route of prime relevance, for instance, must include evaluation of routes present in situ and liable to be adapted so as to serve the main itinerary and to form an integrated set of road infrastructures apt to ensure superior transport performances.

It can be also considered to concentrate the transport demand on alternative modes of transport, capable to ensure rapid, frequent and safe transfers of people and resources, and less onerous in terms of soil consumption and landscape degradation. If re-use or upgrade of present routes is not adequate to meet needs of prime relevance, or if its costs or its burdens on landscapes are estimated too onerous, it is proper to individuate roads or media to be constructed: functions, end-points, scale of routes and performance standards are to be defined. Moreover, it is required to individuate a set of land uses or of features considered susceptible to be disrupted by the proposed transport functions. The final step consists in the individuation of a corridor and of an area of influence, i.e., a belt of land apt to enclose road spaces and sequences of spaces and land uses apt to tie motion and landscape.

This process must be the base for a rigorous plan of transportation. Instances and proposals must be evaluated, mended and adopted in a regional transport plan, conceived as an adaptive document, meant to set a broader vision and a clear indication of priorities.

Once defined the aims and criteria of a profound revision of transport plots, a subsequent procedure must focus on re-use and upgrade of single roads.

A second stage must focus on definition of a sequence of services and urban functions of broader scope to be located along road edges. A punctual investigation of traversed sites is required in order to point out land uses, traditions and ecological factors. A concept of vision as defined by Alexander [13] is a proper tool, in order to investigate a place to point out features required in a specific moment and in a precise place. Moreover, participation is required in order to deduce the image of the place, its perceived order, its references and sacred sites [14].

A consequent step focuses on a fabric of transverse routes and on a plot of minor functions apt to sustain users streams and refers to a concept of roads as a permeable medium meant to encourage people to discover a place and to nurture its routines.

It is required to propose an abacus of modules (number and size of lanes, for cars and/or for public transport means, size of belts reserved for rails, promenades or routes for soft mobility, footbridges, pipelines, conducts for rain drainage, etc.), meant to adapt a road to its renovated functions, to suture opposite road sides, to preserve edges and to sustain consolidated land uses.

A subsequent step must focus on a precise definition of scale, purposes, amenities and location of transport nodes. Since a node is a gate open on a site, its position must emanate from a tern of conditions: it is proper to avoid the onset of movements so intense to saturate local roads and impair uses and sensitive features: it is proper to locate a node close to access 
routes; it is required to define a set of minor routes meant to reinforce a given node and to improve its performances in terms of service provided to adjacent areas.

A transit node is to be considered as provider of urban features, apt to foster a profound regeneration of a broader area [4]. It is also required to define type and location of intersections so as to suture local routes and main itineraries.

Subsequent steps are aimed at defining a mosaic of spaces apt to structure edges and residual surfaces as a porous border and as a device apt to preserve, to bind and to engage a vast range of scales, of practices and of interactions. Time is to be engaged as a medium, in order to enable a gradual adaptation of restored spaces to site-specific forces [3], but also in order to convert edges in a dual space capable to respond to contingent conditions, to sustain specific and periodic forms of motion and to admit a broad range of uses and practices, in periods of rest or of minor scope of "infrastructural flows" [11].

Roads can be conceived as modular adaptive devices, composed of a permanent spine, binded to removable units and spaces conceived as non permanent scenarios. The output is a plot, a set of features and rules. Final stages must structure proposed concepts and programs. A first step focuses on a rigorous road upgrade: measures are elaborated in order to adapt sections, curvatures, profiles and borders. It is also required to structure borders and perceptual relations among users and traversed places. A second step consists in a precise design of transport nodes, of road intersections and of transverse sutures. A subsequent step tends to design urban public services of larger scale, located along road edges.

The final step focuses on road edges and on design of adaptive spaces, scenarios and references and spots. The proposed procedure, however, is unusual. Routes and transport devices are managed by several entities, often in competition with each other: a single institution is presumed to be reluctant to adjust its offer in order to integrate different transport services. In order to overcome divisions and to transform transport systems in an organic structure, nodes, as gates open on sites, should be the focus of an advanced transport governance [7]. Even within a constant confrontation with transport lines operators and supra-local public institutions, users are to detain a pre-eminent role in defining purposes and aims of a profound revision of transport tissues.

\section{Roles}

In the United Kingdom, the Road Traffic Reduction Act [15] proposes and structures involvement of local agencies as actors demanded to estimate and evaluate present and future transport demand, to propose measures intended to reduce car transit, to promote alternative modes of transport, in order to foster fruition of urban features and to reduce burdens on traversed sites, as a part of policies founded on cooperation among diverse levels of government and various agencies, and on a basis of mutual concordance among aims of transport plans and distribution of land uses, defined as a main concern by the PPG13 [16].

As a consequence, local agencies, (municipalities, metropolitan area and unions of municipalities) can be considered as representative institutions of a region. Regions refer to a specific social fabric and define a precise user base. A public institution designates one of its departments or a specific team to evaluate needs and required movements so as to define a precise demand. Moreover, it defines proper models of node, and defines node operators. Local agencies and nodes operators can be coincident. In a second stage, local agencies supervise definition of strategies of revision and upgrade of transport fabrics, and definition of land uses and functions to be promoted along road edges. It is also required, to local agencies, to promote and to regulate participation of residents and investors in processes meant to plan and implement transformation and regeneration of road sides and 
residual spaces. Node operators, also aided by a specific design team define Strategies and define for each road a set of modules and components apt to implement proposed strategies. Node operators are also required to supervise design of road spaces and of road furniture.

Moreover, the node operators could be required to bear part of the costs, for instance in order to moderate fares for future users or if potential user bases are considered too scarce to produce a profit or to justify elevated operative costs. As a result node operators need to find funds, encouraging participation of private partners - e.g. promoting the reuse of road edges - and accessing funds provided by regional, national, or supra-state agencies. A model for policies apt to promote a sensible transformation of road edges can be deduced from the Barnier-Dupont Act on reinforcement of environment protection [17]: since boulevards are conceived as tools meant to preserve built scenarios, vast belts of land, presumed to span from 75 to 100 meters from road edges, are preserved as a space apt to enclose uses and practices related to site vocations and defined by a POS (plan of occupation of surfaces) conceived as outcome of a debate among diverse actors, and of contributions of diverse disciplines.

In the case of minor, remote, urban centers, it could be considered to promote unions of municipalities meant to claim the management of secondary roads or of major roads, deprived of a status of route of prime relevance, by more performant itineraries. Reuse, regeneration and management of minor neglected roads must tend to create a fabric of routes apt to promote rapid and safe movements, in order to merge multiple poles into an urban network. Nodes and urban centers could be connected to large scale services and define a broader user base apt to sustain urban-scale services, such as high schools, theaters, hospitals. This structure can be enhanced by providing a system of mass transport, such as bus or minibus lines.
Super ordinate entities, e.g., cantons, regions, Lander or federal governments, need to act as supervisors, to mediate among involved partners, to promote and regulate participation of investors, to guide and encourage research and to support local entities. Super ordinate entities are also required to evaluate priorities and impacts of a proposed measure on close areas and on routes and transportation plots. Requests and demand for routes and nodes, presented by local entities, must be evaluated by sopra-local entities, in order to individuate needs of prime relevance, to combine near and analogous instances and to promote integrated solutions. Adopted requests and instances are enclosed in a preparatory document. Strategies are evaluated by super-local agencies, amended and enclosed in an organic and adaptive transport plan. Moreover, the super ordinate institution is to allocate funds and cooperate with the node operators to set procedures to ensure access to funds provided by the national government or unions of states.

Transport lines operators are supposed to plan and implement the measures envisaged to standardize and upgrade the transport system: they must therefore integrate the proposals and the priorities contained in the transport plan, and then draft a precise program of interventions, including actions aimed at construction of new routes, and at maintenance, renovation and upgrade of available ones.

Moreover, models and procedures proposed focus on a cooperation at diverse levels among road designers, landscape designers, sociologists, planners, artists, agronomists, geologists, and on a constant and fecund contribution of investors, users, local institutions and residents. As a consequence, a modular design team, composed according to concerns and purposes of single stages, is formed. Design teams are required to conduct a profound investigation of a region, to point out its fabric of sensitive relations, to support local agencies, to mediate among diverse actors and diverse instances, 
to define strategies for an upgrade of transport fabrics and to promote an idea of roads as devices meant to mold landscapes.

Final steps, focused on reuse and regeneration of margins as adaptive and transient figures of public space, require and urge for diverse design and management strategies founded on a participation of residents, future users and investors.

\section{Conclusions}

Landscape is to be considered as a lens in order to understand urban transformations and as a medium in order to mold and structure urban realms: a concept of infrastructure as landscape is nodal in order to order, sustain and combine a broad range of interactions and streams of resources and information, to engage diverse scales and paces, to use and include time and to individuate areas of influence as proper realms for more fecund strategies. The Buffalo Bayou Promenade designed by the SWA (Sasaki, Walker and Associates) group and the Parc Nus de la Trinitat in Barcellona, by Battle y Roig, can be considered as reference cases: an advanced proposal of regeneration of road spaces, focused on reuse and conversion of edges, fringe spaces, voids, subverts a concept of road as monofunctional service space, and enunciates an idea of infrastructure as a mosaic of diverse scenarios, as a linear landscape of diverse spaces, land uses, ecotopes. Residual neglected spaces become resources, field for design strategies set to increase sense and relevance of transport structures as frame and spine, of urban landscapes and as core and focus for urban routines. Edges and samples of void spaces are recovered and converted so as to enclose open spaces, to sustain and to order softer forms of motion, to structure sutures among points divided by road spaces, to restore depleted ecotopes, to create fecund habitats and to manage ecological processes.

Moreover, amount and magnitude of urban concerns increase responsibilities of municipalities and precipitate a trend of decentralization of provision and infrastructure, in order to enable local agencies to respond and to manage interactions and forces related to urban functions. It is required to define policies founded on participation of diverse local agencies, investors and users, on contributions of diverse levels of government, diverse interests, and of a broad set of disciplines. Landscape designers must act as mediators, in order to amend, modulate and combine diverse instances, and intend to propose and define a set of diverse canons and concerns as a basis for advanced policies, and for promotion of a diverse concept of infrastructural spaces.

\section{References}

[1] Muller, S. 2013. "The Next Generation Of Infrastructure.” Scenario Journal 3. Accessed December 11, 2014. http://www.scenariojournal.com.

[2] Olmo, C. 2001. "La Città e le sue Storie (The City and its stories).” In La Città del XXI Secolo-Lezioni di Storia Urbana (The European City of the XXI Century-Lectures of urban history), edited by Mazzeri, C. Milano: Skira, 1726. (in Italian)

[3] Belanger, P. 2013. "Landscape Infrastructure; Urbanism beyond Engineering.” Ph.D. thesis, Wageningen University.

[4] Department of the Environment, Transport and the Regions. 1997. "Road Traffic reduction Act.” Department of the Environment, Transport and the Regions. Accessed December 11, 2014. http://www.legislation.gov.uk.

[5] Hung, Y. Y. 2013. "Landscape Infrastructure: Systems of Contingency, Flexibility and Adaptability.” In Landscape Infrastructure, edited by The Infrastructure Research Initiative at SWA. Basel: Birkhauser, 14-9.

[6] Olivetti, A. 1959. Il Cammino della Comunità (The Path of the Community). Ivrea: Edizioni di Comunità. (in Italian)

[7] Alexander, C., Ishikawa, S., Silverstein, M., Jacobson, M., Fiksdahl-King, I., and Angel, S. 1977. A Pattern Language: Towns, Building, Construction. New York: Oxford Press.

[8] Marshall, S. 2004. "Building on Buchanan: Evolving Road Hierarchy for Today's Streets-Oriented Design Agenda." In Proceedings of the European Transport Conference 2004 (CD-ROM).

[9] Waldheim, C. 2006. "Landscape As Urbanism." In The Landscape Urbanism Reader, edited by Waldheim, C. New York: Princeton Architectural Press, 35-54.

[10] Smets, M., and Shannon, K. 2010. The Landscape of Contemporary Infrastructure. Rotterdam: Nai Publishers. 
[11] Robinson, A. 2013. "Moderating Infrastructural Flows to Create Open Space.” In Landscape Infrastructure, edited by The Infrastructure Research Initiative at SWA. Basel: Birkhauser, 36-41.

[12] Eppell, V. A. T., McClurg, B. A., and Bunker, J. M. 2001. "A Four Level Road Hierarchy for Network Planning and Management.” In Proceedings of the 20th Australian Road Research Board Conference. Accessed April 24, 2014. http://eprints.qut.edu.au/2349/1/2349.pdf.

[13] Alexander, C., Neis, H., Anninou, A., and King, I. 1987. A New Theory for Urban Design. New York: Oxford Press.

[14] Lynch, K. 1960. The Image of the City. Cambridge: The MIT (Massachussets Institutes of Technology) Press.

[15] Smets, M., and Kagner, K. 2013. "Contemporary
Infrastructure: An Interview With Marcel Smets." Scenario Journal 3. Accessed December 11, 2014. http://www.scenariojournal.com.

[16] Department for Communities and Local Government. 2011. "Planning Policy Guidance 13: Transport." Department for Communities and Local Government. Accessed December 11, 2014. http://www.nationalarchives.gov.uk.

[17] Legifrance. 2012. Loi No. 95-101 du 2 Février 1995 Relative au Renforcement de la Protection de L'Environnement (Act 95-101 on Strengthening the Protection of the Environment). Legigrance. Accessed September 10, 2014. http://www.legifrance.gouv.fr. (in French) 